\title{
Location of hot spots in integrated circuits by monitoring the substrate thermal-phase lag with the mirage effect
}

\author{
Xavier Perpiñà,, ${ }^{1, *}$ Josep Altet, ${ }^{2}$ Xavier Jordà, ${ }^{1}$ Miquel Vellvehi, ${ }^{1}$ and Narcís Mestres ${ }^{3}$ \\ ${ }^{1}$ Institut de Microelectrònica de Barcelona IMB-CNM (CSIC), Campus de la Universitat Autonoma \\ de Barcelona, 08193 Bellaterra, Barcelona, Spain \\ ${ }^{2}$ Departament d'Enginyeria Electrònica, Universitat Politècnica de Catalunya, Barcelona 08034, Spain \\ ${ }^{3}$ Institut de Ciència de Materials de Barcelona ICMAB (CSIC), Campus de la Universitat Autonoma \\ de Barcelona, 08193 Bellaterra, Barcelona, Spain \\ *Corresponding author: xavier.perpinya@imb-cnm.csic.es
}

Received May 12, 2010; accepted June 21, 2010;

posted July 9, 2010 (Doc. ID 128313); published July 30, 2010

\begin{abstract}
This Letter presents a solution for locating hot spots in active integrated circuits (IC) and devices. This method is based on sensing the phase lag between the power periodically dissipated by a device integrated in an IC (hot spot) and its corresponding thermal gradient into the chip substrate by monitoring the heat-induced refractive index gradient with a laser beam. The experimental results show a high accuracy and prove the suitability of this technique to locate and characterize devices behaving as hot spots in current IC technologies. () 2010 Optical Society of America
\end{abstract}

OCIS codes: $350.5340,120.4630,120.5710,120.5050,120.6810$.

The scaling-down of microelectronic technologies has enabled an entire system to be integrated on a single chip (system-on-a-chip, SoC). A drawback of these high integration levels is the loss of observability that it entails, since only a few nodes are electrically accessible from the outside. Sensing temperature for locating hot spots provides additional observability in current integrated circuits (ICs) in several scenarios, such as IC failure analysis [1] , system debugging [2], and evaluation of the fabrication process dispersion [3].

In this framework, optically based off-chip thermometries [4] have traditionally sensed the thermal map on the chip topside (frontside approach). However, metal or passivation layers in current IC technologies may strongly or totally attenuate the sensed physical magnitudes [4], making it more difficult to locate the hot spot. Several techniques have been proposed to overcome these challenges, e.g., interferometric measurements [5], backside thermal imaging [6] , or die perimeter scanning [7]. The situation is more restrictive when a packaged IC is intended to be studied-there is no access through the chip's frontside or backside. It is in this situation that die perimeter scanning permits carrying out the hot-spot location [7].

In this Letter, hot spots are located by sensing the heat flux within the IC substrate. It can be measured by traversing the IC substrate with an IR laser probe $(\lambda=1310 \mathrm{~nm})$ at a given depth (inspection depth), thereby avoiding the metal and passivation layers placed over the silicon (IC perimeter scanning) [8]. When the laser beam passes through the substrate, it experiences a deflection proportional to the heat flux found along its trajectory, since a refractive index gradient is thermally induced (mirage effect) [8]. In previous works, the hotspot location was found by processing the amplitude of the vertical and horizontal components of the laser beam deflection as a function of the lateral coordinate $[\underline{7}, 9]$. The location was determined when maximum and null (minimum) values were obtained for the vertical and horizontal deflection signals, respectively. However, this strategy presents a drawback, which concerns the sensitivity of amplitude measurements to experimental error sources, as will be proven in this work. As a solution, we report how, by processing the phase lag between the power excitation waveform and the deflection signals $(\phi)$, a faster and more accurate hot-spot location is achieved.

Figure 1 represents a single metal-oxide-silicon field effect transistor (MOSFET) device or MOS transistor in an IC acting as a hot spot and the thermal field that it generates. When the MOS is activated with a unipolar

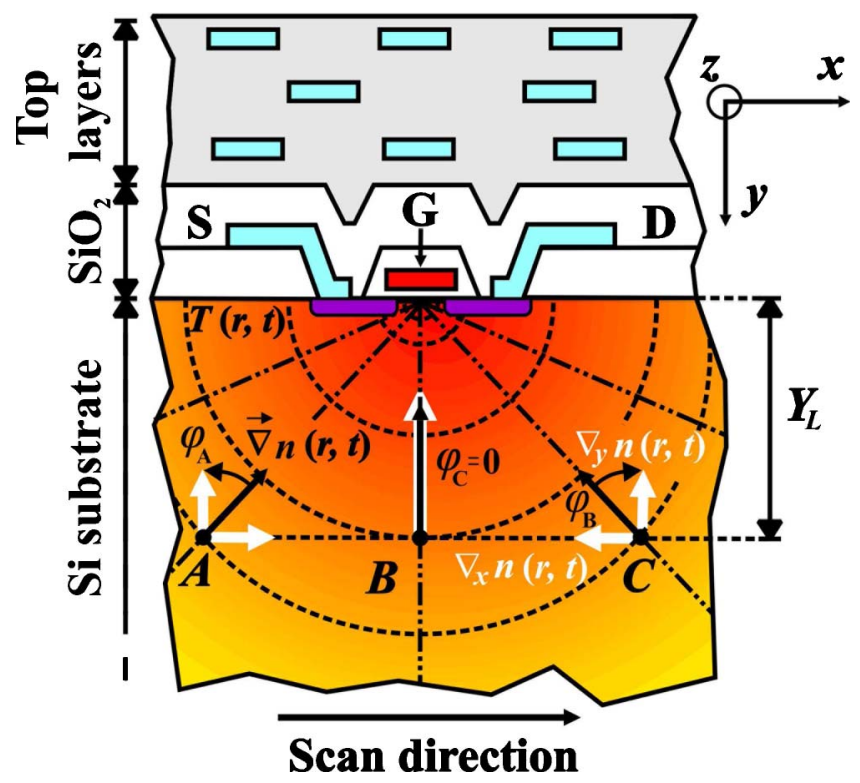

Fig. 1. (Color online) Thermal phenomena induced by an MOSFET dissipating heat within the IC substrate when a lateral scan is performed with a laser beam (points $\mathrm{A}, \mathrm{B}$, and $\mathrm{C}$ ). The direction of the radial unit vector $\hat{u}_{r}$ (black double point-dashed lines) is also highlighted. 
power pulse train and the induced hot spot is small compared to the semiconductor dimensions, the temperature field $(T(r, t))$ can be assumed to be radial in spherical coordinates (see Fig. 2, black dashed lines), and only odd harmonics will be detected in the IC substrate [10]. Besides, the IC substrate can be set as a semi-infinite thermal medium by selecting the suitable working frequency value $f_{\text {heating; }}$; i.e., $f_{\text {heating }} \gg D_{\alpha} \cdot\left(l^{2} \cdot \pi\right)^{-1}$, where $D_{\alpha}$ and $l$ are the substrate thermal diffusivity and thickness, respectively. Under these conditions, the different harmonics of the $T(r, t)$ and, consequently, their associated temperature gradients are confined within the IC substrate [10]. As a result, each harmonic $2 k+1$ of the thermally induced refractive index gradient $\vec{\nabla} n_{2 k+1}(r, t)$ obeys the following equation outside the power generation region $[\underline{7,} \underline{8,10]}$ :

$$
\begin{aligned}
\vec{\nabla} n_{2 k+1}(r, t)= & -A_{2 k+1}(r) \\
& \cdot \sin \left(2 \pi(2 k+1) f_{\text {heating }} t+\psi(r)\right) \hat{u}_{r},
\end{aligned}
$$

where $A_{2 k+1}(r)$ and $\hat{u}_{r}$ are the amplitude of $\vec{\nabla} n_{2 k+1}(r, t)$ and the radial unit vector in spherical coordinates, respectively. $\psi(r)$ is the thermal phase lag and writes as

$$
\psi(r)=\arctan \left(\left(r / d_{p}\right) \cdot\left(1+r / d_{p}\right)^{-1}\right)-r / d_{p},
$$

and $d_{p}$ is the diffusion length defined as

$$
d_{p}=\sqrt{D_{\alpha} \cdot\left(\pi \cdot(2 k+1) \cdot f_{\text {heating }}\right)^{-1}} .
$$

Let us consider the horizontal and vertical components of $\nabla n(r, t)\left(\nabla_{x} n(r, t)\right.$ and $\nabla_{y} n(r, t)$, respectively) at the points labeled A and B in Fig. 1. These points are placed in the same inspection depth $Y_{L}^{-}$and are located in such a way that their angular coordinate $\varphi$ fulfills the condition $\varphi_{\mathrm{A}}=-\varphi_{\mathrm{B}}$. According to Fig. $1, \vec{\nabla} n_{2 k+1}(r, t)$ may be written in terms of the harmonics corresponding to $\nabla_{x} n(r, t)$ and $\nabla_{y} n(r, t)\left(\nabla_{x} n_{2 k+1}(r, t)\right.$ and $\nabla_{y} n_{2 k+1}(r, t)$, respectively) as

$$
\begin{aligned}
& \nabla_{y} n_{2 k+1}(r, t)=-\left\|\vec{\nabla} n_{2 k+1}(r, t)\right\| \cdot \cos (\varphi), \\
& \nabla_{x} n_{2 k+1}(r, t)=\left\|\vec{\nabla} n_{2 k+1}(r, t)\right\| \cdot \sin (\varphi) .
\end{aligned}
$$

Focusing on the $\nabla_{x} n(r, t)$ at points A and B (see Fig. 1) and taking into account the symmetry of the case, the amplitude of $\nabla_{x} n(r, t)$ is the same [see Eq. (5)], but there is a change of sign due to the term $\sin (\varphi)$ that appears in Eq. (5). This is true as long as $\varphi_{\mathrm{A}}=-\varphi_{\mathrm{B}}$ is verified. At point $\mathrm{C}$, it is observed that $\varphi_{\mathrm{C}}=0$, thus, $\nabla_{x} n(r, t)$ nulls. When $\vec{\nabla} n(r, t)$ is sensed following the scan direction defined in Fig. 1, it is expected to detect an abrupt transition in the phase lag of $\nabla_{x} n(r, t)\left(\Delta \phi_{x}\right)$ when the point $\mathrm{C}$ is crossed, since $\sin (\varphi)$ modulates $\psi(r)$ [see Eq. (5)]. As a rule of thumb, the hot spot is precisely located at the lateral coordinate $x$ that corresponds to $\Delta \phi_{x} / 2$.

This new approach has been verified with the same IC used in $[\underline{7}, \underline{9}]$ to compare different hot-spot detection methods. The specific IC is sized $3075 \mu \mathrm{m} \times 2350 \mu \mathrm{m} \times$
$500 \mu \mathrm{m}$ and was developed to study heat-coupling effects in complementary metal-oxide-semiconductor (CMOS) circuits. Figure 2(a) shows the detail of four MOS transistors that may behave as hot spots. These devices are sized $20 \times 1.2 \mu \mathrm{m}^{2}$ and can dissipate up to $28 \mathrm{~mW}$. The square indicates the location of the MOS transistor activated to generate the hot spot, and the arrow highlights the scan direction of the laser beam.

In the performed experiments, the MOSFET has been activated with a unipolar power pulse train with $f_{\text {heating }}=$ $2120 \mathrm{~Hz}\left(d_{p}=116 \mu \mathrm{m}\right.$ for $\left.k=0\right)$ that injects into the chip a heat flux of $1 \mathrm{~mW} \mu \mathrm{m}^{-2}$. The scan has been performed at $Y_{L}=180 \mu \mathrm{m}$ (verifying $Y_{L}>d_{p}$ ), and the MOSFET has been oriented in such a way that its channel is perpendicular to the laser beam propagation direction [see Fig. 2(a)], sensing a $1.2 \mu \mathrm{m}$ hot spot. The vertical and horizontal components of the beam deflection have been detected at the exit of the IC substrate by a self-developed deflection monitoring system (a four-quadrant photodiode, I/V converters, and a postprocessing circuitry) [11]. This system provides electrical signals that are proportional to the laser spot displacement onto the fourquadrant photodiode [11], directly providing information about $\nabla_{x} n_{2 k+1}(r, t)$ and $\nabla_{y} n_{2 k+1}(r, t)$. Afterward, the amplitude and phase lag of the first harmonic $(k=0)$ corresponding to each deflection signal have been determined with a lock-in amplifier.

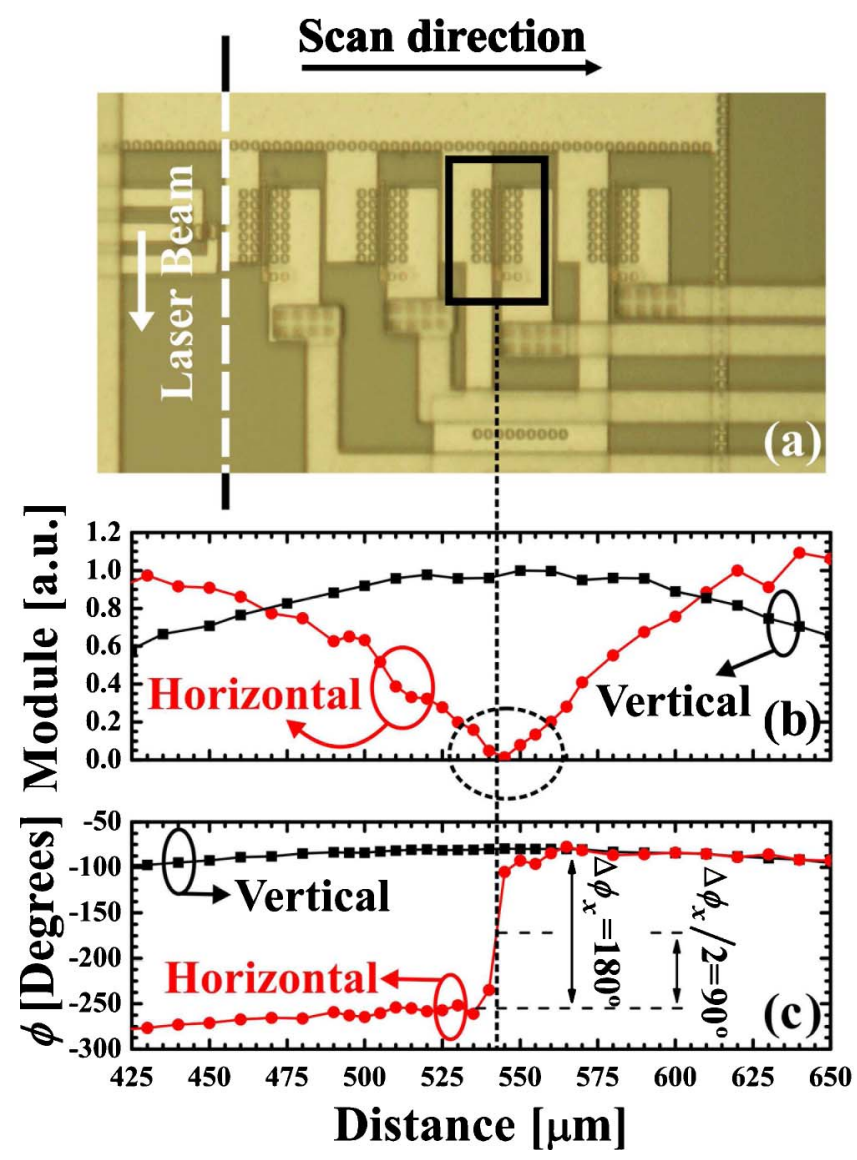

Fig. 2. (Color online) (a) Photograph of the inspected IC indicating the activated MOS, the laser beam sense, and the scan direction. (b) Amplitude and (c) $\phi$ corresponding to $k=0$ of the horizontal and vertical beam deflection as a function of the lateral coordinate $x\left(f_{\text {heating }}=2120 \mathrm{~Hz}\right)$. 
Figures 2(b) and 2(c) depict the amplitude (module) and phase $\overline{\operatorname{lag}(\phi)}$ for both deflection signals, respectively. Data are represented as a function of the lateral IC coordinate. Figure 2(b) shows that the hot-spot lateral coordinate can be asserted by finding either the maximum of the vertical deflection [7] or the null value (minimum) of the horizontal deflection [9] , as previously stated. Figure 2(c) proves that the lateral position of the device acting as a hot spot is indicated by the transition in the horizontal phase deflection $\left(\Delta \phi_{x}\right)$ : the hot spot is located at $x=528.5 \mu \mathrm{m}$, corresponding to $\Delta \phi_{x} / 2=$ $90^{\circ}$. One can realize from Figs. 2(b) and 2(c) that determining $\phi_{x}$ has major advantages in comparison with the other extracted information, as it is less sensitive to the experimental error sources. In fact, there are three main experimental error sources: the electronic noise related to the deflection monitoring system [11], the positioning stage accuracy, and the heat-spreading effect. First, the electronic noise mostly affects the amplitude of the horizontal deflection, as it is expected to nullify beneath the hot spot [9]. In contrast, $\phi_{x}$ experiences an abrupt $\Delta \phi_{x}$ beneath the position of the hot spot in such a way that small phase oscillations do not have any effect on the measurement accuracy. Second, the positioning stage accuracy affects all measurements negatively. It is necessary to have a positioning stage with a spatial accuracy similar to the dimensions of the heat source. However, $\Delta \phi_{x}$ allows interpolating the correct position of the heat source more easily than in the other situations. Third, the heat spreading significantly influences the vertical deflection signals increasing the width of the maxima observed in the amplitude and phase (more pronounced as $Y_{L}$ increases).

In conclusion, hot spots in ICs are located following a new strategy based on laterally accessing the chip substrate with a laser beam. The thermal phase lag between the power dissipated by a MOSFET and the thermally induced refractive index gradient inside the IC substrate is extracted from the laser beam deflection (mirage effect). The experimental results show how a heat source is located at inspection depths higher than 2 orders of magnitude $\left(Y_{L}=180 \mu \mathrm{m}\right)$ of their lateral dimensions $(1.2 \mu \mathrm{m})$, almost reaching a submicrometer accuracy.

This work has been partially supported by the Consejo Superior de Investigaciones Científicas (CSIC) (under contract "Junta para la Ampliación de Estudios," JAEDoc) and the Spanish Ministry of Science and Innovation (research programs THERMOS TEC2008-05577, RUE CSD2009-00046, and TERASYSTEMS TEC2008-01856).

\section{References}

1. M. Pedram and S. Nazarian, Proc. IEEE 94, 1487 (2006).

2. S. A. Bota, J. L. Rossello, C. de Benito, A. Keshavarzi, and J. Segura, IEEE Des. Test Comput. 23, 414 (2006).

3. J. Jaffari and M. Anis, IEEE Trans. Comput.-Aided Des. Integr. Circuits Syst. 27, 1027 (2008).

4. J. Altet, W. Claeys, S. Dilhaire, and A. Rubio, Proc. IEEE 94, 1519 (2006).

5. J. Altet, M. A. Salhi, S. Dilhaire, A. Syal, and A. Ivanov, Electron. Lett. 39, 1440 (2003).

6. G. Tessier, M. Bardoux, C. Boué, C. Filloy, and D. Fournier, Appl. Phys. Lett. 90, 171112 (2007).

7. X. Perpiñà, J. Altet, X. Jorda, M. Vellvehi, J. Millan, and N. Mestres, IEEE T. Comput. Aid. D. 29, 1142 (2008).

8. X. Perpiñà, X. Jordà, N. Mestres, M. Vellvehi, P. Godignon, J. Millán, and H. von Kiedrowski, Meas. Sci. Technol. 15, 1011 (2004).

9. X. Perpiñà, X. Jordà, J. Altet, M. Vellvehi, and N. Mestres, J. Phys. D Appl. Phys. 42, 012002 (2009).

10. H. S. Carslaw and J. C. Jaegger, Conduction of Heat in Solids (Clarendon, 1986).

11. X. Perpiñà, X. Jordà, M. Vellvehi, J. Millán, and N. Mestres, Rev. Sci. Instrum. 76, 025106 (2005). 